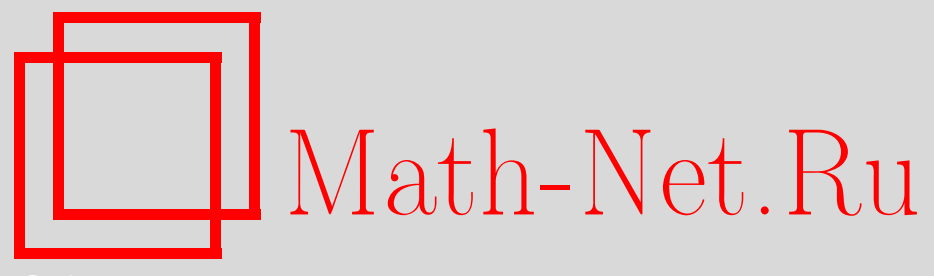

В. П. Маслов, Решение парадокса Гиббса в рамках классической механики (статфизики) и кристаллизация газа $C_{60}$, Матем. заметки, 2008, том 83, выпуск 5, 787-791

DOI: https://doi.org/10.4213/mzm4725

Использование Общероссийского математического портала Math-Net.Ru подразумевает, что вы прочитали и согласны с пользовательским соглашением http://www . mathnet.ru/rus/agreement

Параметры загрузки:

IP: 54.196 .121 .252

26 апреля 2023 г., 15:48:30

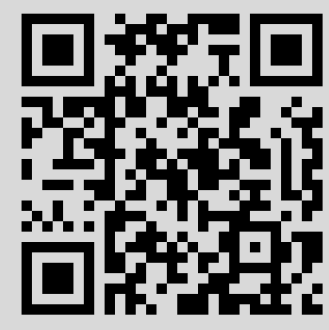




\section{Решение парадокса Гиббса в рамках классической механики (статфизики) и кристаллизация газа $C_{60}$}

\section{В. П. Маслов}

В своей статье [1] на с. 68 я привожу пример из сферы экономики в пользу теории exchangeability (instead of independence condition). В этом же ключе, на мой взгляд, надо произвести ревизию "гипотезы Гиббса о термодинамическом равновесии", которое основывается (см. [2]) на свойстве независимости, приводящем к теореме умножения вероятностей. Именно эта гипотеза и приводит к распределению Гиббса, а оно опровергается парадоксом Гиббса, т.е. по сути дела, математическим контрпримером к этой гипотезе.

$\mathrm{C}$ математической точки зрения, речь идет об упорядоченной и неупорядоченной выборке [3]. Козлов показал, что гипотеза эргодичности, на которой основываются попытки доказать распределение Гиббса, также неверна. Он нашел замечательный класс - множество Пуанкаре. По-видимому, это множество может быть использовано и для "динамического стремления" к приводимому ниже распределению.

Пусть $E_{0}$ - энергия, связанная со средней поступательной скоростью молекул в жидкости, находящейся в равновесном состоянии газ-жидкость. ${ }^{1}$ Эта скорость много меньше средней скорости молекул газа. Нельзя сказать, что эти скорости и, соответственно, энергия "конденсата" равны нулю.

Мы рассмотрим модель равновесия двух фаз “газ-жидкость” (без примесей!), в которой будем учитывать только поступательную скорость молекул в "конденсате" (жидкости) и нормировать интегралы по $p$ и $q$ будем на фазовый объем, ограниченный уровнем энергии, определяемым средней скоростью молекул в конденсате (жидкости). Он, разумеется, увеличивается при увеличении температуры.

Перейдем теперь к выводу распределения типа Бозе-Эйнштейна в классической механике.

Пусть $N$ - число свободных частиц с гамильтонианом $p^{2} / 2 m$ в объеме $\Omega, p=\left(p_{x}, p_{y}, p_{z}\right)$, и пусть энергия $N$ частиц ограничена константой $\mathscr{E}_{N} \gg 1$, т.е. $\mathscr{E}_{N} / E_{0} \rightarrow \infty$. Найдем величину $b$ из соотношения

$$
\int \frac{p^{2}}{2 m}\left(e^{b \cdot p^{2} / 2 m}-1\right)^{-1} d p=\mathscr{E}_{N}, b \rightarrow 0 \quad \text { при } \quad \mathscr{E}_{N} \rightarrow \infty .
$$

Определим через

$$
\mathscr{N}=\frac{1}{\left(m E_{0}\right)^{3 / 2}} \int\left(e^{b \cdot p^{2} / 2 m}-1\right)^{-1} d p
$$

число частиц, "насыщающих" объем $\Omega$, и $N \geqslant \mathscr{N}$.

Разобьем пространство импульсов $p$ на конечное число областей

$$
E_{l} \leqslant \frac{p^{2}}{2 m} \leqslant E_{l+1}, \quad l=0, \ldots, s-1, \quad E_{0}>0, \quad E_{s}=E ; \quad p \in \mathbb{R}^{3} .
$$

Производем неупорядоченную выборку с возвращением $N_{l}$ из $N$ частиц (шаров) в "ящик" $E_{l} \leqslant p^{2} / 2 m \leqslant E_{l+1}$ при условии

$$
\sum_{i=1}^{N} \frac{p_{i}^{2}}{2 m} \leqslant \mathscr{E}_{N} .
$$

Пусть $\rho_{E_{l}}-$ плотность числа частиц в интервале меньшем или равном $E_{l}: \rho_{E_{l}}=$ $\sum_{j=1}^{l} N_{i} / \mathscr{N}$.

$\mathrm{B}$ нижеприводимой теореме $\mathrm{P}$ есть лебегова мера фазового объема, определенного в скобках (3), относительно всего объема (2).

\footnotetext{
1Эта модель приводится для наглядности. Более реальна ситауция, когда жидкая фаза отсутствует, как для $C_{60}$, и когда рассматривается равновесие газ-твердое тело.
}

(C) В. П. МАСлов, 2008 
Теорема 1. Имеет место соотношение

$$
\mathrm{P}\left(\left|\rho_{E_{l}}-\int_{p^{2} / 2 m \leqslant E_{l}}\left(e^{b \cdot p^{2} / 2 m}-1\right)^{-1} d p / \int\left(e^{b \cdot p^{2} / 2 m}-1\right)^{-1} d p\right| \geqslant N^{-1 / 3+\varepsilon}\right)=O\left(\mathscr{N}^{-k}\right),
$$

где $k$ - любое целое, $\varepsilon>0$ - сколь угодно малое.

Все $N-\mathscr{N}$ частиц лежат на уровнях не выше $E_{0}$, если $N-\mathscr{N}>\mathscr{N}^{2 / 3+\varepsilon}$.

ДокАзАтЕльство. Теорема следует из общей теоремы о бозе-распределении [1]. Уточнение оценок приводится в [4], [5]. Выберем такое разбиение, что $E_{l+1}-E_{l}=E_{0}$ и $\left(E_{l+1}-E_{l}\right) / E>\mathscr{N}^{-1 / 4}$. Тогда

$$
E_{l}=E_{0}(l+1) .
$$

Пусть $N_{l}$ - неупорядоченная выборка с возвращением в ячейку $E_{l+1}-E_{l}$. Иными словами, неупорядоченная выборка с возвращением из $N$ шаров по ячейкам (“энергетическим ящикам")

$$
\operatorname{dim} \Omega \int_{E_{l}}^{E_{l+1}} \frac{p^{2}}{2 m} d p=\frac{2}{5}\left(E_{l+1}^{5 / 2}-E_{l}^{5 / 2}\right) \operatorname{dim} \Omega
$$

приводит к соотношению

$$
\sum_{l} N_{l} E_{l} q_{l} \leqslant \mathscr{E}_{N}, \quad q_{l} \cong C\left[l^{1 / 2}\right]
$$

$C$ - константа.

Это значит, что для "кратностей вида $\left[l^{1 / 2}\right]$ ” для “чисел заполнения" $N_{l}$ выполняется теорема 1 работы [5], а именно, выполнено следующее.

Положим $\Delta N=\mathscr{N}^{2 / 3+\varepsilon}$, где $\varepsilon$ - достаточно малое положительное число. Существуют такие постоянные $C_{k}, k=1,2, \ldots$, что для любого $l \in \mathbb{Z}_{+}$справедлива оценка

$$
P\left(\left|\sum_{l=1}^{s}\left(N_{l}-\bar{N}_{l}\right)\right|>\Delta N\right) \leqslant C_{k} \mathscr{N}^{-k},
$$

где $\bar{N}_{l}=q_{l} /\left(e^{b l}-1\right), l=1,2, \ldots .\left(q_{l}\right.$ в нашем случае равно $\left.q_{l}=\left[l^{1 / 2}\right]\right)$.

Так как $\rho_{E_{l}}=\sum_{j=1}^{l} N_{j} / \mathscr{N}$, то отсюда следует, что после применения формул Эйлера-Маклорена к ряду

$$
\sum_{l=l_{0}}^{s} \bar{N}_{j}=\sum_{l=l_{0}}^{s} q_{l} \frac{1}{e^{b l}-1}
$$

получим

$$
\sum_{l=l_{0}}^{s} q_{l} \frac{1}{e^{b l}-1} \sim \int_{l_{0}}^{s} \frac{x^{1 / 2}}{e^{b x}-1} d x .
$$

Отсюда, сделав замену $x=p^{2} / 2 m$ и поделив (5) на $\mathscr{N}$, получим утверждение теоремы.

Чтобы отличить классический случай от квантового, мы будем заменять термин "выпадение в конденсат" термином "кластеризация". Частицы при $N>\mathscr{N}$ как будто бы кластеризуются, "склеиваются", и уже кластеры движутся с энергией $E_{0}$, и если находятся в поле тяжести, то "падают на дно" (см. ниже).

Теперь рассмотрим случай $N<\mathscr{N}$, т.е. число частиц меньше насыщающего числа. В этом случае перед экспонентой появляется фактор $c>1+\varepsilon, \varepsilon-$ сколь угодно малое, независящее от $b$ положительное число.

Пусть при заданных $\mathscr{E}_{N}$ и $N$ константы $b$ и $c$ вычисляются из условий

$$
\begin{aligned}
\int \frac{p^{2}}{2 m}\left(c e^{b \cdot p^{2} / 2 m}-1\right)^{-1} d p & =\mathscr{E}_{N}, \\
\int\left(c e^{b \cdot p^{2} / 2 m}-1\right)^{-1} d p & =N .
\end{aligned}
$$


Справедлива теорема:

Теорема 2. Имеет место соотношение

$\mathrm{P}\left(\left|\rho_{E_{l}}-\int_{p^{2} / 2 m \leqslant E_{l}}\left(c e^{b \cdot p^{2} / 2 m}-1\right)^{-1} d p / \int\left(c e^{b \cdot p^{2} / 2 m}-1\right)^{-1} d p\right| \geqslant \sqrt{\frac{\ln N}{N}} \psi(N)\right)=O\left(N^{-k}\right)$,

где $k$ - любое иелое.

ДокАзАТЕЛьство аналогично доказательству теоремы 1.

Отсюда

$$
\frac{N}{\mathscr{N}}=\frac{\int x^{1 / 2} /\left(c e^{x}-1\right) d x}{\int x^{1 / 2} /\left(e^{x}-1\right) d x}
$$

и при $N / \mathscr{N} \rightarrow 0, c \rightarrow \infty$, что приводит к распределению Максвелла. При этом фактор в этом распределении таков, что парадокса Гиббса уже не может возникнуть. Это, впрочем, было очевидно из первоначальной постановки.

Для классического идеального газа, т.е. в случае $\mathscr{N} / N \gg 1$, выполняется соотношение

$$
\frac{P}{n \theta}=1
$$

где $P$ - давление газа, $n=N / V$ - концентрация, $\theta$ - температура в энергетических единицах, $\theta=k T$, где $k$ - постоянная Больцмана, $T$ - абсолютная температура (в кельвинах). Это уравнение состояния идеального классического газа.

Для $\mathscr{N} / N>1$ получаем

$$
\frac{P}{n \theta}=\frac{\zeta(5 / 2)}{\zeta(3 / 2)} \approx \frac{1.341}{2.612} \approx 0.523
$$

где $\zeta$ - функция Римана.

Однако достичь состояния $\mathscr{N} / N>1$ экспериментально трудно, так как наличие примесей (например, аэрозолей в водяном паре) приведет к соотношению (12). Поэтому в эксперименте можно лишь утверждать, что каким бы чистым он ни был, добиться соотношения

$$
\frac{P}{n \theta}<\frac{\zeta(5 / 2)}{\zeta(3 / 2)}
$$

невозможно, если не учитывать отталкивающий потенциал (см. замечание в конце статьи; ср. с понятием фазового перехода нулевого рода [6]). В этом смысле можно сказать, что состояние

$$
1>\frac{P}{n \theta} \geqslant 0.523
$$

метастабильно, а при $P / n \theta<0.523$ - неустойчиво. Отметим, что сверхтекучее состояние также метастабильно.

Применимость распределения типа Бозе-Эйнштейна и существование кластеризации для классического газа могут быть проверены в следующем эксперименте. Необходимо создать перенасыщенный газ фуллеренов, не содержащий примесей. Чтобы фуллерены не оседали на стенках сосуда, стенки должны быть покрыты графеном (лучше натянутым на твердый каркас-подложку). ${ }^{2}$ При внесении в сосуд, например, куска ткани, алюминия, пластмассы избыток молекул фуллерена осядет на материи - произойдет конденсация, при этом установится равновесие между фуллереном в твердой и газообразной фазах. Количество осевшего на материи фуллерена будет зависеть от того, насколько перенасыщен газ фуллеренов. Экспериментальные данные можно сравнить с тем количеством, которое предсказывают полученные в данной работе формулы для

\footnotetext{
${ }^{2}$ Если газ осаждается на стенках, то необходимо подкачивать недостающее число частиц.
} 
числа насыщения объема частицами и числа частиц в конденсате. Если атомно-силовой микроскоп (AFM) может различить более мелкие кластеры, то энергия этих кластеров может быть больше, чем $E_{0}$.

Пусть $T$ - температура насыщения в объеме $\Omega$. Понижаем температуру до $T_{0}$ так, чтобы

$$
\frac{R\left(T-T_{0}\right)}{E_{0}}=c\left(\frac{R T_{0}}{E_{0}}\right)^{s},
$$

где $1 / 4<s \leqslant 1 / 2, c$ - константа. Тогда конденсат возникает при

$$
E_{1} \cong c E_{0}\left(\frac{R T_{0}}{E_{0}}\right)^{2-4 s} .
$$

Отсюда, число частиц перенасыщенного "пара" при температуре $T_{0}$ будет равно

$$
N_{0}=\int_{0}^{\infty} \frac{1}{e^{\beta_{0} \cdot p^{2} / 2 m}-1} d p,
$$

где $\beta_{0}=\left(R T_{0}\right)^{-1}$, число лишних (добавленных) частиц

$$
\int_{0}^{\infty} \frac{1}{e^{\beta \cdot p^{2} / 2 m}-1} d p-N_{0}
$$

где $\beta=(R T)^{-1}$. Однако число частиц после окончательного выпадения в конденсат, т.е. число частиц вне конденсата (число частиц насыщенного пара), равно

$$
\int_{\left(R T_{0}\right)^{-s}}^{\infty} \frac{1}{e^{\beta_{0} \cdot p^{2} / 2 m}-1} d p
$$

а значит, меньше чем за вычетом "лишних частиц". Таким образом происходит скачек числа частиц, т.е. фазовый переход 1-го рода, в отличие от перехода к перенасыщенному пару (фазовый переход 3-го рода). Значит, метастабильное состояние перенасыщенного пара, когда только начнется появление зародышей кристаллов, постепенно перейдет в стабильное состояние насыщенного пара со скачком числа частиц и энтропии.

Предлагаемая экспериментальная схема может быть также использована для решения технологической задачи - создания тканей, пропитанных молекулами фуллерена. Степень пропитки ткани при этом можно регулировать, определяя степень перенасыщенности газа фуллеренов.

Аналогично теоремам 1 и 2 доказывается нижеследующая теорема.

Пусть функция Гамильтона $H(p, q)=p^{2} / 2 m+V(q)$, где $p, q \in \mathbb{R}^{3}, V(q) \leqslant|q|^{6}$ при $q \rightarrow \infty$, и пусть при заданных $\mathscr{E}_{N}$ и $N$ константы $b>0$ и $c \geqslant 1$ вычисляются из условий

$$
\begin{aligned}
\int \frac{p^{2}}{2 m}\left(c e^{b\left(p^{2} / 2 m+V(q)\right)}-1\right)^{-1} d p d q & =\mathscr{E}_{N}, \\
\int\left(c e^{b\left(p^{2} / 2 m+V(q)\right)}-1\right)^{-1} d p d q & =N, \\
\int\left(e^{b\left(p^{2} / 2 m+V(q)\right)}-1\right)^{-1} d p d q & =\mathscr{N} .
\end{aligned}
$$

Теорема 3. Имеет место соотношение

$$
\begin{array}{r}
\mathrm{P}\left(\left|\rho_{E_{l}}-\int_{p^{2} / 2 m+V(q) \leqslant E_{l}}\left(c e^{b\left(p^{2} / 2 m+V(q)\right)}-1\right)^{-1} d p d q / \int\left(c e^{b\left(p^{2} / 2 m+V(q)\right)}-1\right)^{-1} d p d q\right|\right. \\
\left.\geqslant \frac{\ln N}{\sqrt{N}} \psi(N)\right)=O\left(N^{-k}\right),
\end{array}
$$

где $k$ - любое целое, $N \leqslant \mathscr{N}$. 
При $N>\mathscr{N}$ вместо $N$ в неравенстве и оценке (18) нужно поставить $\mathscr{N}$. Разность $N-\mathscr{N}$ кластеризуются, а $c=1$.

Если есть внешнее поле (например, поле земного притяжения), а также вычислен потенциал отталкивания между молекулами фуллерена [7], то можно написать уравнение для одетого потенциала, зависящего от $b$ (т.е. от температуры), и получить поправки вида $(15)-(17)$, где $V(q)$ зависит от $b$ в том же объеме $\Omega$.

Интеграл по $p$ и $q$ по всему пространству при $c=1$ даст зависимость $\mathscr{N}$ от $b$ и $m$, уточняющую (14), которая может быть проверена на эксперименте.

Предъявим интегральное уравнение для одетого потенциала для газа фуллерена:

$$
U(x, y, z)=-m g z+\varepsilon \beta^{-3 / 2} \int_{0.35 \text { нанометров }}^{\infty}\left[\left\{\left(\frac{d}{r}\right)^{43}-\left(\frac{d}{r}\right)^{9}\right\} F(\beta U(\xi, \eta, \zeta)) d \xi d \eta d \zeta\right],
$$

$g$ - ускорение силы тяжести, $d=0.96$ нанометра, $\varepsilon / R=6150 \mathrm{~K}$ (кельвина),

$$
r=\sqrt{(x-\xi)^{2}+(y-\eta)^{2}+(z-\zeta)^{2}} ;
$$

$F(\beta U)$ - функция вида

$$
F(\beta U)=\beta^{3 / 2} \int_{-\infty}^{\infty} \frac{d p_{x} d p_{y} d p_{z}}{e^{\beta U} e^{\beta \cdot|p|^{2} / 2 m}-1}=\frac{2}{\sqrt{\pi}} e^{-\beta U} \Gamma\left(\frac{3}{2}\right) \Phi\left(e^{-\beta U}, \frac{3}{2}, 1\right),
$$

где $\Phi$ - специальная функция, задаваемая формулой

$$
\Phi(\alpha, \nu, \mu)=\sum_{n=0}^{\infty}(\mu+n)^{-\nu} \alpha^{n}
$$

$m$ - масса $C_{60}, \beta=1 / R T_{0}, T_{0}$ - температура в кельвинах, $R$ - константа Больцмана; радиус молекулы фуллерена равен 0.35 нанометра.

\section{СПИСОК ЦИТИРОВАННОЙ ЛИТЕРАТУРЫ}

[1] V.P. Maslov, Russ. J. Math. Phys., 14:1 (2007), 66-95. [2] В. В. Козлов, Tenловое равновесие по Гиббсу и Пуанкаре, Современная математика, Ин-т компьютерных исследований, М.-Ижевск, 2002. [3] А.Н. Ширяев, Вероятность, кн. 1: Элементарная теория вероятностей. Математические основания. Предельные теоремы, МЦНМО, М., 2004. [4] В.П. Маслов, В.Е. Назайкинский, Матем. заметки, 83:2 (2008), 232-263. [5] В. П. Маслов, В. Е. Назайкинский, Матем. заметки, 83:3 (2008), 381-401. [6] В. П. Маслов, Квантовая экономика, Наука, М., 2006. [7] Л. Н. Якуб, Физика низких mемператур, 19 (1993), 726.

\section{В. П. Маслов}

Поступило

Московский государственный 20.03.2008 университет им. М. В. Ломоносова E-mail: v.p.maslov@mail.ru 